\title{
Analytical Approach for Loss Minimization in Distribution Systems by Optimum Placement and Sizing of Distributed Generation
}

\author{
Surbhi Bakshi ${ }^{1 \text { a }}$, Tilak Thakur $^{2}$, Rintu Khanna ${ }^{3}$ \\ ${ }^{1}$ Research Scholar, Deptt. of Electrical Engg, PEC University of Technology, Chandigarh, India \\ ${ }^{2}$ Professor, Deptt. of Electrical Engg, PEC University of Technology, Chandigarh, India \\ ${ }^{3}$ Associate Professor, Deptt. of Electrical Engg, PEC University of Technology, Chandigarh, India
}

\begin{abstract}
Distributed Generation has drawn the attention of industrialists and researchers for quite a time now due to the advantages it brings loads. In addition to cost-effective and environmentally friendly, but also brings higher reliability coefficient power system. The DG unit is placed close to the load, rather than increasing the capacity of main generator. This methodology brings many benefits, but has to address some of the challenges. The main is to find the optimal location and size of DG units between them. The purpose of this paper is distributed generation by adding an additional means to reduce losses on the line. This paper attempts to optimize the technology to solve the problem of optimal location and size through the development of multi-objective particle swarm. The problem has been reduced to a mathematical optimization problem by developing a fitness function considering losses and voltage distribution line. Fitness function by using the optimal value of the size and location of this algorithm was found to be minimized. IEEE14 bus system is being considered, in order to test the proposed algorithm and the results show improved performance in terms of accuracy and convergence rate.
\end{abstract}

Keywords: Distributed Generation, Particle Swarm Optimization, Optimal Sizing, Solar.

\section{Introduction}

Distributed Generation involves the applying of little generators, scattered throughout an influence system, to produce the electrical power required by electrical customers. Appeared, because of its low cost of electricity for non-traditional growing interest, it is environmentally friendly, due to increased awareness of emission control, which has become an important factor [1], [2], [3]. This environment is a benefit and investment in distributed generation in particular solar, wind, cogeneration (CHP). There are a variety of factors, such as improving work efficiency, environmental benefits and better transmission congestion management contribute to its popularity. The main idea here is to have a large number of DG units which are close to the load centre, rather than increasing the capacity of the main power plant. Various techniques can help achieve DG as renewable energy, such as solar energy, wind energy, tidal energy, biomass energy, they are more common address of "green energy." Micro turbines, diesel engines, gas turbines, fuel cells from other additions to the list, even though they are not so-called energy "green" form. Stirling engine and the engine further extend the list of reciprocating engines [4], [5], [6] Recently, wind energy has gained huge popularity and all other forms of renewable energy [7] to tough competition.

DG can be defined as the generation and transmission system, wherein the generator system is not in direct contact to the main transmission grid, but to the distribution network. It has economic, environmental and technical reliability many advantages. Reducing losses due to transmission and distribution caused by reduced transmission equipment costs, saving energy as a result of lower electricity prices. Environment is also obtained from the sound level of emission reductions in pollution and greenhouse gas levels lower. Wide range of advantages such as reduced line losses, increase peaking, improving the system's voltage profile and ultimately leads to higher power quality. This leads to transmission and distribution and grid reinforcement ease congestion. Sometimes it is also used to provide a separate system, 
remote application with the required power to the load in. Therefore, it is necessary to find the best location and optimal size of distributed power.

Best location DGs were found only in the reduced basis [8] losses. Some power flow algorithm has been raised several researchers in order to find the best size DG, in all load buses [9] [10]. Wang Nehrir analysis shows the preferred placement targeting dangerous goods minimum loss. Attempts have been made by the Chiradeja benefit from reducing feeder loss distribution in the radial direction, wherein the load is concentrated. Meta-heuristic algorithms have been used by some researchers to find new DG units [11-15] in the best position. Genetic algorithms have been used to DG placement and loss reduction. Multi-objective evolutionary algorithms have been used by the Botticelli and Ghiani to solve this problem. Nara et.al, investigate DG optimal location and size by using tabu search algorithm.

This paper identifies the use of multi-objective particle swarm optimization optimum value size and placement of the additional DG units i.e. the size of the bus and the best location in order to obtain the ultimate goal. Multiobjective optimization including seeking the optimal value, so that the voltage and the loss is the best way to optimize the weight and importance depends on various factors such as the type of power required quality and economic requirements, etc. [15] for obtaining data in IEEE bus 14. In addition, the power supply is integrated and distributed using Newton - Raphson method.

This paper is organized as follows. The problem formulation to determine the optimal size of DGs at the selected locations is defined in Section II and proposed methodology in section III. Then, the effectiveness of the proposed method is verified with the simulation results in Section IV. Finally, the conclusions are given in Section V.

\section{Problem Formulation}

Problems Optimizing Distributed power generation unit is configured in the form of formulated optimization problem taking into account the cost function of the voltage and the transmission line formed by the real power loss. This problem can be represented mathematically in Equation 12:

$$
\text { minimize } f(x)=w 1 \sum_{k=0}^{n} \operatorname{loss}+w 2 * \sum_{k=0}^{m} \operatorname{los}(1-V i)^{\wedge} 2
$$

Here, ' $m$ ' is the number of the bus and " $\mathrm{n}$ " is the number of lines in the system. The problem is tested for IEEE-14 bus system. The IEEE-14 bus system consists of $6 \mathrm{PV}$ bus out with bus number 1 is connected with a maximum capacity of slack bus in which the main generators is connected. Newton Raphson technique is used to calculate Power flow in the network, as the analytical solution of the problem is impossible.

\section{Proposed methodology}

Newton iterative method is used in this work for trend analysis. Each bus is calculated from the trend in terms of active and reactive power. In addition, line losses in each row in the bus system are also calculated. Goal of this article is to join an additional DG to reduce losses on the line. Additional equipment will meet the increased load demand. An objective function is dependent on the distribution line losses and voltage formed.

The objective function is improved using particle swarm optimization and constraint handling of reflection is also minimized. As the problem is converted into a constrained optimization problem; the original PSO is modified to handle infeasible solutions. Also the size of the problem is a digital estimation and allocation problem is integer estimation and therefore the solution is checked for validity in the search space. Improving the voltage profile and reducing the loss of multi-objective function is converted to a minimization problem.

The position of the unit is initialized in IEEE-14, system bus 1-14 for a random integer value. All solutions are looking to check the feasibility of space and initialization process is repeated until a feasible solution. Each particle is updated according to the rules and checks the validity of the group, and only those particles that are feasible will remain unchanged, and the rest will be rejected. This process continues until the maximum or minimum error criterion iteration conditions are achieved.

\section{Results and Discussions}

The problem defined above has been simulated for an IEEE 14 bus system. 100 particles were considered and simulations were done for 30 iterations. All the simulations were done in MATLAB R2013a in a 64 bit system with $2.3 \mathrm{GHz}$ processor and $4 \mathrm{~GB}$ RAM.

Divergence among the particles as the iteration progresses can be depicted in Figure 1. 


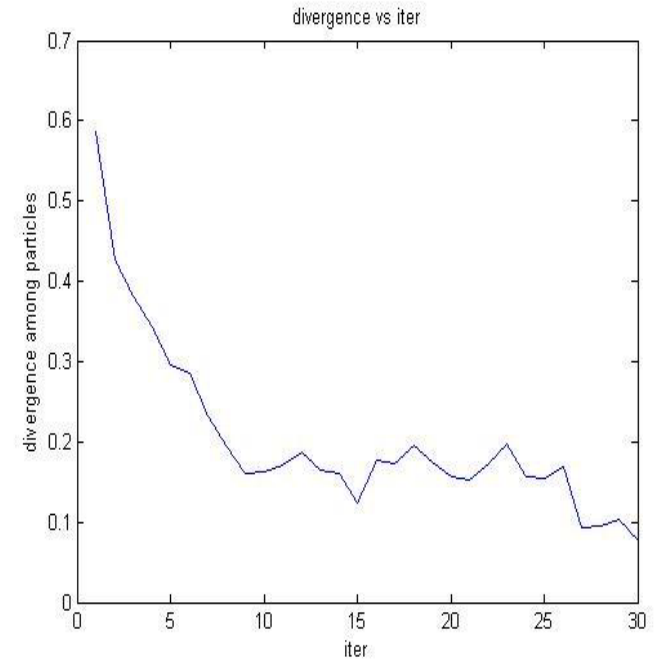

Figure 1. Plot of Divergence of Particles It is observed that the divergence decreases with progress in iteration. The reason behind is the convergence of particles. As the Swarm algorithm searches in the search space for optimal values, particles start converging towards a common value and divergence among particles being diminishing.

A pictorial representation of the particles is provided in Figure 2 and it presents a real view of how the particles move in the search space. As it can be observed that, the particles begin randomly within the specified range but finally converge to a single point overcoming all the local minimas and maximas.

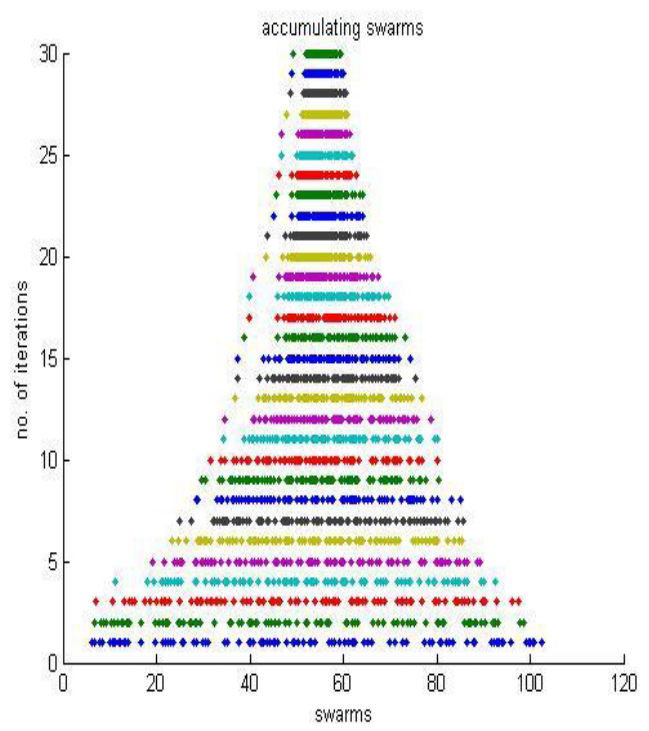

Figure 2. Plot of convergence of particles
The table 1 illustrate the optimal results of 14 bus systems. Table 2 illustrate voltages after simulation on each bus of 14 bus system

Table 1. Showing optimized values

\begin{tabular}{|l|l|}
\hline Bus System & 14 \\
\hline Optimal Bus No & 14 \\
\hline $\begin{array}{l}\text { Loss(MW) without } \\
\text { DG }\end{array}$ & 4.2 \\
\hline $\begin{array}{l}\text { Loss (MW) } \\
\text { With DG }\end{array}$ & 3.3063 \\
\hline Optimal Size (kW) & 32.1839 \\
\hline
\end{tabular}

Table 2. Voltage (14 bus system)

\begin{tabular}{|c|c|}
\hline Bus No & Voltage \\
\hline 1 & 1.06000000000000 \\
\hline 2 & 1.04500000000000 \\
\hline 3 & 1.01000000000000 \\
\hline 4 & 1.01319412327445 \\
\hline 5 & 1.01657458089408 \\
\hline 6 & 1.07000000000000 \\
\hline 7 & 1.04569913905268 \\
\hline 8 & 1.08000000000000 \\
\hline 9 & 1.03052499541293 \\
\hline 10 & 1.02991793536347 \\
\hline 11 & 1.04613111278885 \\
\hline 12 & 1.05325955541164 \\
\hline 13 & 1.04663639983200 \\
\hline 14 & 1.01927185909764 \\
\hline
\end{tabular}

\section{Conclusions}

An effective solution to the problem of optimal sizing and location of DG units using a multi-objective Particle Swarm Optimisation technique was proposed. On further analysis it is found that the optimal values of these parameters can reduce losses and improve voltage profile significantly. The algorithm converges after around 30 iterations and is computationally efficient too. The algorithm is designed such that it increases the exploration capability initially and avoids being trapped in local minimas and maximas. The fitness on the basis of which the optimisation algorithm is based is a multi dimensional function and hence the solution obtained is only a pareto-optimal solution. 


\section{References}

1. J. De La Ree, V. Centeno, J. S. Thorp and A. G. Phadke, "Synchronized phasor measurement applications in power systems," IEEE Transactions on Smart Grid, vol. 1, no.1, pp. 20-27, (2010)

2. A. G. Phadke and J. S. Thorp, Computer Relaying for Power System. Second edition, Chichester: John Wiley \& Sons Ltd, (2009)

3. P. Ju, C. Qin, F. Wu, H. Xie and Y. Ning, "Load modeling for wide area power system," International Journal of Electrical Power \& Energy Systems, vol. 33, no. 4, pp. 909-917, (2011)

4. Z. Q. He, Z. Zhang, W, Chen, O. P. Malik and X. G. Yin, "Wide-area backup protection algorithm based on fault component voltage distribution," IEEE Transactions on Power Delivery, vol.26, no. 4, pp. 2752- 2760, (2011)

5. A. G. Phadke and J. S. Thorp, Synchronized Phasor Measurements and Their Applications. Springer verlag, (2008)

6. S. H. Horowitz and A. G. Phadke, "Third zone revisited," IEEE Transactions on Power Delivery, vol.21, no.1 pp.23-29, (2006)

7. X. Tai, D. Marelli, E. Rohr and M. Fu, "Optimal PMU placement for power system state estimation with random component outages," International Journal of Electrical Power \& Energy Systems, vol.51, pp.35-42, (2013)

8. T. Ackerman, G. Andersson and L. Söder, "Distribution Generation: a definition". Electric Power System Research 57 pp. 195-204., (2001)

9. Chiradeja P., "Benefits of Distributed Generation:A line loss reduction analysis", Transmission and
Distribution Conference and Exhibition: Asia and Pacific, 2005 IEEE/PES .

10. Kejun Qian, Cheng K. Zhou, Yue Yuan,Xiaodan Shi and Allan M., "Analysis of the Environmental Benefits of distributed Generation",Power and Energy Society General Meeting- Conversion and Delivery of Electrical Energy in the 21st century,2008 IEEE.

11. Gil, H.A.and Joos,G.,"Models for Quantifying the Economic Benefits of distributed Generation", IEEE Transactions on Power Systems, Vol.23, pp. 327 335, (2008)

12. Chiradeja P.and Ramakumar, R.," An approach to quantify Technical Benefits of Distributed Generation", IEEE transactions on Energy, Vol. 19, No. 4, pp.764-773, (2004)

13. Waseem, I, Pipattanasomporn M. and Rahman, S.,"Reliability Benefits of Distributed Generation as Back up source",,IEEE Power and Energy society General meeting, PES-2009, Publication Year: 2009 , Page(s): $1-8$.

14. Momoh, J.A., Yan Xia, and Boswell, G.D.," An approach to determine Distributed Generation benefits in power networks", 40th NA Powerr Symposium, pp. 1-7, (2008)

15. P. Chiradeja and R. Ramakumar, "A review of distributed generation and storage," in Proc. 1998 Frontiers of Power Conf., pp. VIII 1-VIII 